\title{
UM PROJETO DE ECONOMIA CIRCULAR SOB A PERSPECTIVA DO GERENCIAMENTO DE RISCOS: O REAPROVEITAMENTO DE RESÍDUOS SÓLIDOS INDUSTRIAIS.
}

Daiane Rossi (dai-rossi@hotmail.com) - Programa de Pós-Graduação em Engenharia de Produção PPGEP, Universidade Federal do Rio Grande do Sul (UFRGS).

Ricardo Marques Sastre (ricsastre@gmail.com) - Programa de Pós-Graduação em Engenharia de Produção - PPGEP, Universidade Federal do Rio Grande do Sul (UFRGS).

Márcia Elisa Echeveste (echeveste @ producao.ufrgs.br) - Programa de Pós-Graduação em Engenharia de Produção - PPGEP, Universidade Federal do Rio Grande do Sul (UFRGS).

\section{RESUMO}

A abordagem de economia circular contempla práticas que buscam a gestão dos recursos, especialmente melhor aproveitamento de resíduos para gerar valor para a empresa e a cadeia. Entretanto, modelos de negócio baseados nas práticas de economia circular apresentam riscos potenciais envolvendo o meio ambiente, segurança, relações na cadeia, e a viabilidade econômica. Este estudo visa investigar e avaliar os riscos de projeto em uma empresa de reaproveitamento de resíduos sólidos industriais. Para tal analisou-se um projeto de desenvolvimento de produtos de uma empresa recuperadora de resíduos industriais, que atua na cadeia calçadista. O método percorreu fases do gerenciamento de riscos como análise qualitativa e quantitativa dos riscos, com o uso de técnicas como matriz de probabilidade e impacto, viabilidade econômica, análise de sensibilidade e de cenários. Levando em consideração os pilares econômico, ambiental e social do projeto e do negócio, para avaliar a viabilidade deste projeto. Os resultados demonstraram a viabilidade econômica do projeto e as oportunidades para tomada de decisão, além de propostas para o tratamento dos riscos encontrados, podendo ser mitigados, evitados ou explorados.

Palavras chave: riscos; resíduos industriais; economia circular; valor sustentável. 


\section{INTRODUÇÃO}

A geração e destino dos resíduos sólidos, especialmente os industriais, é um problema no Brasil que gera bilhões de toneladas de resíduos ao ano (BRASIL, MMA, 2020). Estes resíduos poderiam passar por outras formas de tratamento antes da disposição final, a destinação ao aterro. Com isto, os governos ampliam a fiscalização da legislação e aumentam os custos de disposição em aterro para as empresas (BRASIL, 2020). Frente a este contexto, configura-se uma oportunidade de utilizar os princípios da economia circular na redução e no aproveitamento dos resíduos.

A economia circular visa o melhor aproveitamento dos recursos e energia sob forma de inovação com mudanças nos modelos de negócios (BOCKEN, 2014; LEWANDOWSKI, 2016). Os modelos de negócio na economia circular diferem da economia tradicional também nos seus riscos. Os riscos incluem oportunidades e ameaças relacionadas aos assuntos econômicos, ambientais e sociais, incluindo a reputação da empresa, o mercado sustentável, a capacidade de inovação e as alterações legais (SCHULTE, 2018).

De acordo com a norma internacional para gerenciamento de riscos, a ISO 31000, os riscos são efeito das incertezas sobre os objetivos da empresa os quais a organização não possui controle (PURDY, 2010). Porém, os riscos não precisam ser considerados positivos ou negativos, mas serem vistos como formas de atingir os objetivos (PURDY, 2010; SCHILLER, 2014). Ao avaliar os riscos e o valor de um negócio é necessário incluir os aspectos econômicos, ambientais e sociais considerando as dimensões da sustentabilidade, dentro da proposição de valor sustentável (HART, 2003; PATALA, 2016).

Os riscos associados ao desenvolvimento de produtos a partir do reaproveitamento de resíduos industriais carecem de dados históricos e geram dúvidas ao consumidor quanto a percepção de valor de seus produtos. Entretanto, modelos de negócio baseados nas práticas de economia circular apresentam riscos potenciais envolvendo o meio ambiente, segurança, relações na cadeia, e a viabilidade econômica. Com a avaliação destes riscos pode-se esclarecer as oportunidades e ameaças envolvidas para a geração de valor no modelo de negócio. Este valor é percebido, tanto internamente como com os gastos evitados com recursos, como externamente quando o mercado permite o retorno econômico gerado pelos produtos. Para o presente estudo, presume-se que o valor sustentável para a cadeia se dá pela ampliação do ciclo de vida do material, maximização do uso dos recursos e eliminação dos resíduos. Com a finalidade de contribuir com este tema, este estudo visa investigar e avaliar 
os riscos de projeto no reaproveitamento de resíduos sólidos industriais, incluindo aspectos ambientais e sociais e econômicos para a proposição de valor sustentável.

\section{REVISÃO TEÓRICA}

O gerenciamento de riscos corporativos busca integrar de forma sistemática todos os tipos de riscos em um portfólio e aos objetivos da empresa, visando oportunidades de crescimento para a organização (SCHILLER, 2014). De acordo com Purdy (2010) as empresas gerenciam os riscos da organização com o uso de métodos para a priorização, mitigação, eliminação ou ainda exploração dos riscos. Quando os riscos são vistos como oportunidades e com a mudança na estratégia de ação sobre eles, pode-se obter benefícios para a organização (PURDY, 2010). Este estudo integra abordagens da literatura sobre o gerenciamento de riscos em projetos e organizações com negócios sustentáveis e economia circular.

\subsection{Análise de riscos em projetos de sustentabilidade}

A integração da sustentabilidade com os objetivos da organização se faz necessária do mesmo modo como no gerenciamento de riscos corporativos, de acordo Schulte (2018). Corroborando, Palousis (2010) descreve que os aspectos relacionados à sustentabilidade devem ser verificados na fase de projeto do produto, pois nesta fase os custos ainda não estão comprometidos. Uma vez que existem riscos financeiros, estratégicos e operacionais nas empresas de manufatura relacionados com a sustentabilidade. Estando de acordo com a proposição de Cagliano (2015) que aponta a avaliação de riscos em projetos, dentro do gerenciamento de riscos tradicional.

\subsection{O valor associado ao reaproveitamento de resíduos}

As empresas passaram a ver na sustentabilidade oportunidades de negócio capazes de diminuir custos e riscos, ampliar mercados através da inovação, simultaneamente gerando valor para a empresa e para o desenvolvimento sustentável (HART, 2003). Uma das oportunidades de criação de valor é a partir dos resíduos, transformando-os em matérias primas para outros produtos e processos e com isto utilizar a capacidade máxima dos recursos (LEWANDOWSKI, 2016; BOCKEN, 2014). A literatura suporta este contexto, Palousis (2010) aborda os processos de reuso e de reciclagem na manufatura ao identificar, avaliar e 
modelar o impacto dos riscos de sustentabilidade nos custos do ciclo de vida do produto. Enquanto Essoussi (2010) avalia o risco e o valor associado com produtos reciclados na percepção dos consumidores. Igualmente, o estudo da IBM (2020) sobre o consumo de produtos sustentáveis demonstra que existe uma predisposição a pagar até 35\% a mais em relação a um produto convencional, mediante a percepção e identificação deste como sustentável.

\section{METODOLOGIA}

\subsection{Descrição do Estudo de Caso}

O estudo de caso descreve a análise de viabilidade de um projeto de um novo produto para uma empresa, com o uso do gerenciamento dos riscos do projeto. A empresa estudada atua no aproveitamento de resíduos da indústria calçadista, utilizando-os como matéria-prima principal de seus novos produtos e na gestão de resíduos na cadeia, promovendo a circularidade por eliminar a disposição destes resíduos. O principal material produzido pela empresa é uma placa laminada de plástico e elastômeros, que é utilizado atualmente em componentes de calçados (CNI, 2019). O projeto avaliado é a produção e comercialização de uma embalagem para calçados com este material. O projeto visa que a empresa seja fornecedora de produto final, como substituto de produto tradicional (caixa de sapatos feita de papelão). As informações sobre o projeto foram coletadas através de entrevista estruturada com um colaborador da empresa e com um responsável pelo projeto das embalagens.

\subsection{Técnicas de Análise de Riscos}

O estudo envolve as fases de gerenciamento de riscos de projetos (PMI, 2008; PURDY, 2010; CAGLIANO, 2015), incluindo aspectos ambientais juntamente com os econômicos para a avaliação de um projeto de economia circular. São abordadas a identificação dos riscos, análise e propostas para o tratamento destes. Cada fase requer a aplicação de técnicas específicas, que são escolhidas de acordo com o nível e qualidade de informação disponível sobre o projeto. As fases da análise de riscos do projeto são demonstradas na Figura 1. 


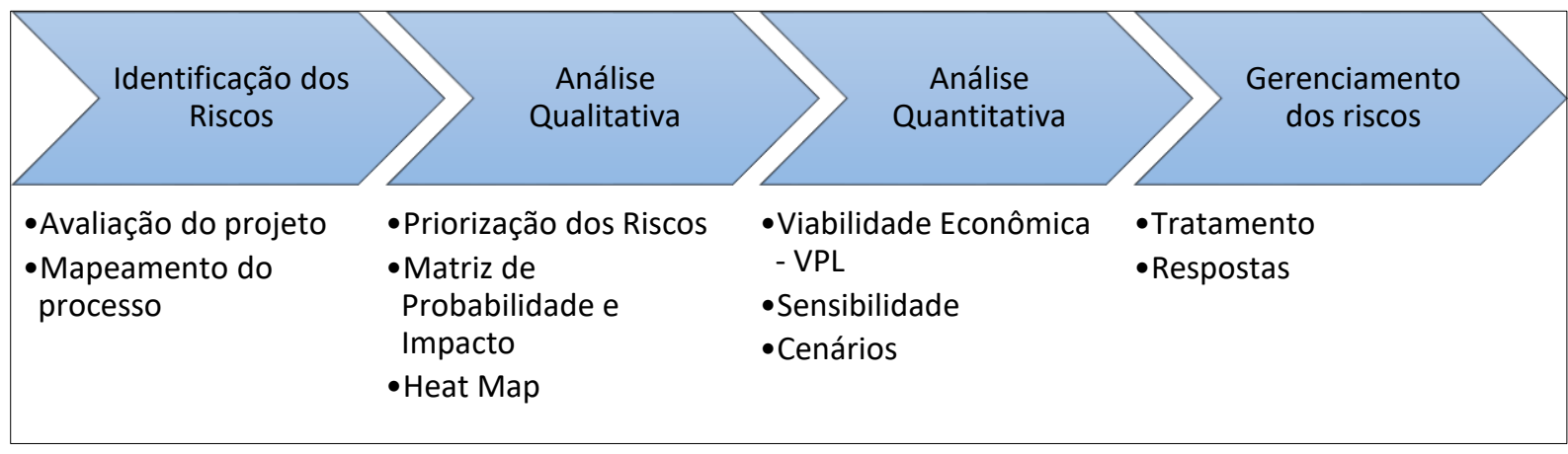

FIGURA 1 - Etapas de análise de riscos do projeto. Fonte: Elaborado pelo autor.

Identificação dos Riscos: nesta etapa é contextualizado e são compreendidas as características e objetivos do projeto do novo produto para a empresa. Com o mapeamento do processo são elencados fatores internos e externos de acordo com a etapa de identificação de riscos do PMI (2008). Com os riscos identificados, faz-se a análise qualitativa destes. Verifica-se a probabilidade de ocorrência e o impacto que pode ser gerado com a concretização destes riscos, baseado em análise de forças e ameaças. Matriz de Probabilidade de impacto: é uma ferramenta de análise qualitativa, auxiliar na categorização e subsequente priorização de riscos em projetos, com a finalidade de atribuir ao risco uma classificação global como baixo, médio ou alto (PMI, 2008), de modo que os de maior gravidade sejam tratados primeiro (CAGLIANO, 2015). Os riscos classificados na matriz de probabilidade e impacto também podem ser representados em um mapa de calor, auxiliando na visualização para a priorização dos riscos a serem tratados.

A etapa de análise quantitativa dos riscos auxilia na geração de respostas para o tratamento destes, com o uso de ferramentas como: Viabilidade econômica que utiliza dados do projeto do processo produtivo, para a criação e análise da planilha de fluxo de caixa, considerando o valor presente líquido (VPL) ao final do prazo estimado. A análise de sensibilidade auxilia a identificação das variáveis críticas do processo, responsáveis pelas maiores variações no valor presente líquido final do fluxo de caixa. A avaliação dos cenários se faz com a combinação das variáveis críticas, em situações hipotéticas, para identificar o impacto no valor presente líquido.

Conjuntamente, as análises qualitativa e quantitativa de riscos contribuem para uma visão sistêmica do projeto, auxiliando o gerenciamento e a tomada de decisão. Com base nos dados levantados nestas etapas, o tratamento dos riscos procura formular resposta aos riscos. As respostas aos riscos ficam a critério do gerenciamento das variáveis críticas que podem tratar 
os riscos como ameaças e os mitigar, ou transferir, ou como oportunidades e aceitar ou explorar (PMI, 2008; CAGLIANO, 2015).

\section{RESULTADOS E DISCUSSÃO}

\subsection{Identificação dos riscos}

Para a avaliação de riscos deste estudo de caso, considerou-se a empresa como futura fornecedora de um novo produto e desenvolvedora de um novo processo de fabricação. Com este intuito, levantou-se os riscos associados ao desenvolvimento do projeto. Os riscos internos elencados estão associados à produção do material enquanto os riscos externos remetem ao mercado e incluem concorrência, aceitação (demanda, valor de venda), matériaprima, e ao meio ambiente como descarte do material final. Os riscos identificados nesta etapa foram: possíveis falhas no processamento, gerando problemas associados a qualidade, o aproveitamento e otimização da área de material, a reciclagem do material, as variações de demanda, o preço de venda, o custo de matéria-prima, a quantidade de matéria-prima disponível, a geração de resíduos.

\subsection{Análise qualitativa dos riscos}

Esta etapa avalia os riscos identificados para sua priorização e ação subsequente baseada na

combinação da probabilidade de ocorrência e o impacto que a concretização deste risco pode trazer. As grandezas de probabilidade e impacto são atribuídas em uma escala subjetiva que varia de 0 a 1 baseadas no mapeamento das atividades aplicados ao objetivo do projeto. A Tabela 1 demonstra os riscos encontrados neste projeto, avaliados de acordo com sua probabilidade de ocorrência e possível impacto.

TABELA 1 - Riscos identificados no projeto com suas probabilidades e impactos.

\begin{tabular}{lcccc}
\hline Riscos & Id & Probabilidade & Impacto & P x I \\
\hline demanda & R3 & 0,6 & 0,9 & 0,54 \\
preço de venda & R4 & 0,6 & 0,8 & 0,48 \\
reciclagem material & R2 & 0,7 & 0,5 & 0,35 \\
aproveitamento do material & R1 & 0,5 & 0,5 & 0,25 \\
custo da matéria prima & R5 & 0,2 & 0,7 & 0,14 \\
resíduos gerados & R6 & 0,3 & 0,3 & 0,09 \\
defeitos & R7 & 0,2 & 0,3 & 0,06 \\
\hline
\end{tabular}




\begin{tabular}{lllll}
\hline quantidade de matéria prima & R8 & 0,1 & 0,3 & 0,03 \\
\hline
\end{tabular}

Os riscos elencados na matriz estão representados em um mapa de calor na Figura 2.

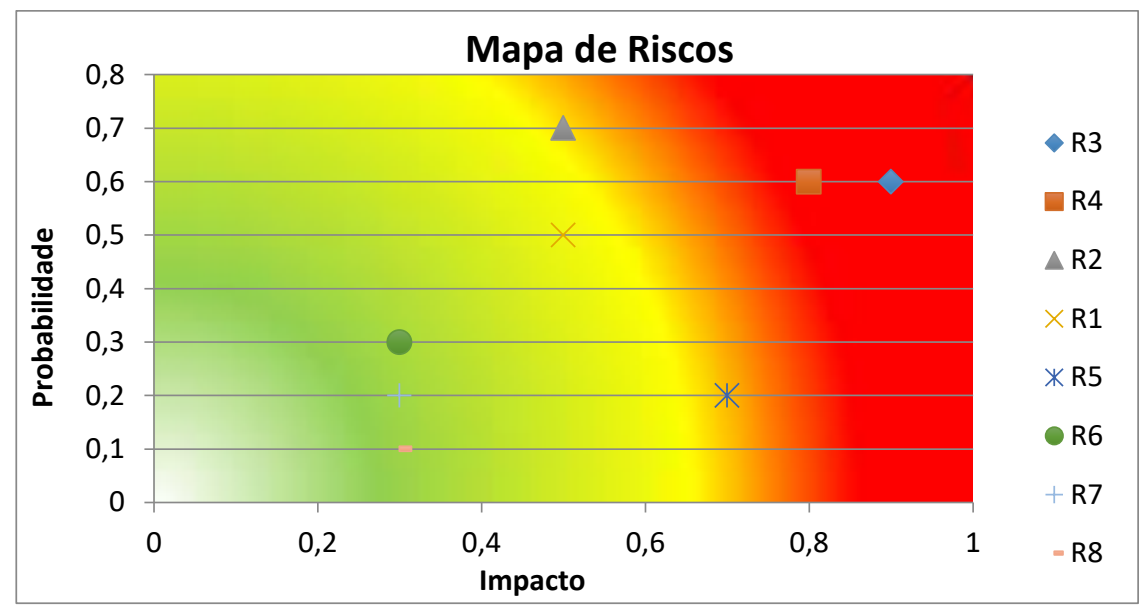

FIGURA 2 - Mapa de calor para os riscos elencados de acordo com a probabilidade e impacto. Fonte: Elaborado pelo autor.

A visualização dos riscos auxilia na priorização, assim, no mapa de calor observa-se que os riscos associados a demanda (R3) e preço de venda (R4) encontram-se em uma região mais crítica no mapa, seguidos do risco associado à reciclagem (R2) e aproveitamento do material (R1) indicando que precisam ser gerenciados e verificada a urgência de tratamento.

\subsection{Análise Quantitativa dos Riscos}

Para a avaliação quantitativa dos riscos, utiliza-se dados do projeto do processo produtivo, necessários para a realização deste. Inclui investimentos iniciais, previsões de custos e receitas e a estimativa de atratividade do projeto, em 10 anos. Esta etapa busca avaliar a viabilidade de oferecer o produto em estudo no mercado. A avaliação quantitativa dos riscos poderá responder se este é competitivo ao mesmo preço do produto padrão.

Para isto, utilizou-se algumas premissas e suposições para o cálculo da viabilidade econômica: o valor estimado para o investimento em equipamentos no processo produtivo foi de $\mathrm{R} \$ 265.000,00$. Os equipamentos avaliados foram: guilhotina, máquina de corte e vinco, impressora e hot stamping, incluídos custos de instalação. $\mathrm{O}$ valor foi baseado nos custos de equipamentos para gráfica usados, consultados em novembro de 2020, e desconsiderado o valor residual do investimento.

Dados de entrada do projeto: prazo do projeto de 10 anos; custo unitário do produto (custos fixos e de produção); preço de venda; demanda; taxa mínima de atratividade do projeto 
(TMA) de 10\%; impostos 21\%; inflação $4 \%$ a.a. A Tabela 2 demonstra os dados de entrada do fluxo de caixa para o projeto da fabricação da caixa de sapatos em material laminado.

TABELA 2 - Dados de entrada para o cálculo de viabilidade econômica do projeto. Fonte: Elaborado pelo autor.

\begin{tabular}{ll}
\hline Projeto Caixa de sapato & \\
Investimento & $\mathrm{R} \$ 265.000,00$ \\
Custo unitário & $\mathrm{R} \$ 4,78$ \\
Impostos & $21 \%$ \\
Custo un. sem impostos & $\mathrm{R} \$ 3,76$ \\
Preço de venda & $\mathrm{R} \$ 5,58$ \\
Demanda mensal & 3.000 \\
TMA & $10 \%$ \\
Inflação & $4 \%$ \\
\hline
\end{tabular}

Os dados sobre custos foram fornecidos pela empresa, e indicam que os custos de processamento, mão de obra, impostos e material geram um produto final com custo unitário de $\mathrm{R} \$ 4,78$. Para a estimativa de receita e fins comparativos, pesquisou-se que uma gráfica concorrente pode oferecer um produto semelhante, em papelão, ao preço de venda unitário de $\mathrm{R} \$ 5,58$. As tiragens da gráfica são de mil unidades. Com estes dados, foi possível criar uma planilha de fluxo de caixa para a avaliação de viabilidade econômica do projeto em estudo. Os dados podem ser observados na Tabela 3.

TABELA 3 - Demonstrativo do cálculo de viabilidade econômica do projeto através do valor presente líquido (VPL). Fonte: Elaborado pelo autor.

\begin{tabular}{|c|c|c|c|c|c|c|}
\hline $\mathbf{T}$ & INVEST & RECEITA INFL & CUSTO INFL & FC AI & IR a pagar & FC DI \\
\hline $\mathbf{0}$ & $-\quad 265.000,00$ & & & - $\quad 265.000,00$ & & $-\quad 265.000,00$ \\
\hline 1 & & $208.915,20$ & $140.774,40$ & $68.140,80$ & $14.309,57$ & $53.831,23$ \\
\hline 2 & & $217.271,81$ & $-\quad 146.405,38$ & $70.866,43$ & $14.881,95$ & $55.984,48$ \\
\hline 3 & & $225.962,68$ & $152.261,59$ & $73.701,09$ & $15.477,23$ & $58.223,86$ \\
\hline 4 & & $235.001,19$ & $158.352,05$ & $76.649,13$ & $16.096,32$ & $60.552,81$ \\
\hline 5 & & $244.401,24$ & $164.686,14$ & $79.715,10$ & $16.740,17$ & $62.974,93$ \\
\hline 6 & & $254.177,28$ & - $\quad 171.273,58$ & $82.903,70$ & $17.409,78$ & $65.493,92$ \\
\hline 7 & & $264.344,38$ & - $178.124,53$ & $86.219,85$ & $18.106,17$ & $68.113,68$ \\
\hline 8 & & $274.918,15$ & - $\quad 185.249,51$ & $89.668,64$ & $18.830,42$ & $70.838,23$ \\
\hline 9 & & $285.914,88$ & - $\quad 192.659,49$ & $93.255,39$ & $19.583,63$ & $73.671,76$ \\
\hline \multirow[t]{5}{*}{10} & & $297.351,47$ & - $\quad 200.365,87$ & $96.985,61$ & $20.366,98$ & $76.618,63$ \\
\hline & & & & & & $\mathrm{R} \$ 318.047,71$ \\
\hline & & & VPL (NO FC) & $\mathrm{R} \$ 137.592,04$ & VPL & R\$ 53.047,71 \\
\hline & & & TIR & $25,91 \%$ & TIR & $19,06 \%$ \\
\hline & & & TIR LIQU & $21,07 \%$ & TIR LIQ & $14,48 \%$ \\
\hline
\end{tabular}


A avaliação dos dados do valor presente líquido e a taxa interna de retorno, demonstrados como valores positivos, indicam que o projeto é viável financeira e economicamente, com os dados de entrada estimados. As variações dos dados de entrada impactam diretamente no cálculo da viabilidade do projeto. Uma ferramenta utilizada para avaliar estas variações é a análise de sensibilidade do projeto, que consiste em variar nominalmente os dados de entrada do projeto e verificar seu impacto no resultado final, neste caso o VPL.

A análise de sensibilidade: As variações utilizadas para esta análise podem ser nominais ou condizentes com a realidade de mercado da empresa. Para o caso avalia-se a variação de $5 \%$ nos custos, podendo chegar ao máximo em $\mathrm{R} \$ 4,02$; variação de demanda de 1.000 unidades por pedido, em lotes e tiragens de impressão; a variação do preço de venda é limitada ao valor que torna o VPL zero, ou acima do preço do produto de mercado. A proposta de um valor ainda maior no preço de venda, com um aumento de $25 \%$ é analisada, devido a pesquisas de mercado que demonstram a propensão dos consumidores em pagarem um valor prêmio por produtos sustentáveis (IBM, 2020).

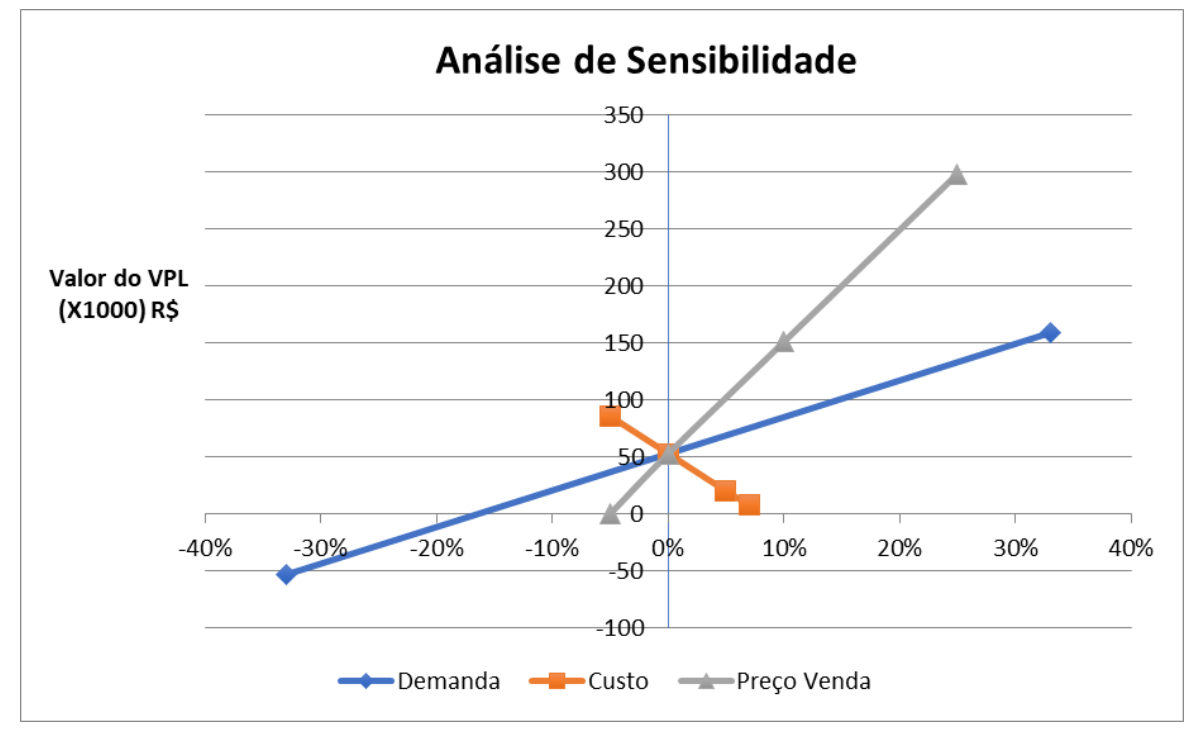

FIGURA 3 - Análise de sensibilidade das variáveis de entrada do projeto, com variação baseada no mercado atual. Fonte: Elaborado pelo autor.

Os resultados dos cálculos destas variações no VPL final do projeto são representados graficamente conforme a Figura 3. A variação mais significativa (com maior inclinação da reta) indica a variável crítica na sensibilidade do projeto. Neste caso o preço de venda é a variável crítica, por ser responsável pela maior alteração do VPL final do projeto. A segunda variável crítica é a variação da demanda e por fim, os custos internos. Os valores limites, que 
mantêm o VPL positivo, para estas variáveis são preço de venda mínimo de $\mathrm{R} \$ 5,28$; custo unitário máximo de $\mathrm{R} \$ 4,06$ e demanda mínima de 2.500 unidades mensais.

Avaliação de cenários: Enquanto a análise de sensibilidade avalia a oscilação de uma variável crítica, mantendo as demais fixas, a análise de cenários avalia as variáveis críticas combinadas. A criação dos cenários se dá com valores maiores ou menores das variáveis críticas, de acordo com as possibilidades e riscos do mercado. As opções de cenários são: pessimista, mais provável e otimista; analisados e demonstrados conforme a Tabela 4.

TABELA 4 - Análise de cenários do projeto. Fonte: Elaborado pelo autor.

\begin{tabular}{cccc}
\hline CENÁRIOS & PESSIMISTA & MAIS PROVÁVEL & OTIMISTA \\
\hline PREÇO & 5,28 & 5,58 & 6,14 \\
CUSTO & 4,02 & 3,76 & 3,57 \\
DEMANDA & 2.000 & 3.000 & 4.000 \\
VPL & $-\mathbf{R} \$ \mathbf{1 1 8 . 2 0 8 , 6 9}$ & $\mathbf{R} \mathbf{5 3 . 0 4 7 , 7 7}$ & $\mathbf{R} \mathbf{3 3 3 . 8 1 5 , 1 6}$ \\
TIR & $-1,84 \%$ & $14,48 \%$ & $34,94 \%$ \\
\hline
\end{tabular}

A interpretação dos cenários demonstra que algumas combinações de riscos podem inviabilizar o projeto, pois impactam no valor do VPL do projeto tornando-o negativo, o que sugere que os riscos identificados precisam ser gerenciados.

\subsection{Gerenciamento dos riscos identificados}

No gerenciamento dos riscos o tratamento é a fase posterior à análise destes. Com os resultados da análise quantitativa percebe-se a necessidade de gerenciar o preço de venda, a demanda e o custo unitário deste projeto. As respostas aos riscos podem ser:

Mitigar os riscos: gerenciar internamente os custos com ajustes de processo para o aproveitamento da placa de material e otimização da área de corte.

Evitar os riscos: não assumir a fabricação da caixa pela empresa, que pode ser um projeto inviável frente a um cenário pessimista, uma vez que o preço de venda, que é um risco externo precisa ser gerenciado avaliando o mercado concorrente e explorando o produto sustentável proposto.

Transferir os Riscos: venda da matéria prima para o atual produtor de embalagens - a viabilidade deste negócio para as gráficas implica em baixos custos de investimento em maquinário, mas em um aumento no custo da matéria prima, que pode ser gerenciado internamente. 
Explorar os Riscos: busca de um novo mercado - atualmente a placa de laminado pode ser oferecida para venda ao preço de $\mathrm{R} \$ 7,46 \mathrm{~m}^{2}$, sem custos adicionais de processamento, que existem na produção da embalagem. O uso da embalagem com logística reversa nos outros elos da cadeia como distribuidores e lojas, evitando a geração de resíduos.

Neste estudo verifica-se que em função dos custos do projeto, que ele é viável em alguns cenários, e avaliando o negócio e a gestão de resíduos que a empresa é capaz de realizar, verifica-se oportunidades de geração de valor dentro da empresa, além do projeto da caixa de sapatos.

\section{CONSIDERAÇÕES FINAIS}

Neste estudo investigou-se e avaliou-se os riscos de projeto em uma empresa de reaproveitamento de resíduos sólidos industriais, incluindo aspectos ambientais e sociais e econômicos para a proposição de valor sustentável. O projeto avaliado foi o de produção e comercialização de uma embalagem de sapatos, desenvolvida a partir de material reciclado. Este projeto é economicamente viável em cenários mais prováveis, mas a concretização de riscos de mercado pode inviabilizá-lo. Diante desta situação, a empresa pode estudar respostas aos riscos levantados. Os riscos vistos como custos remetem a ameaças, e quando associados ao valor como oportunidades; enxergar os riscos como oportunidades de negócios leva a uma resposta de exploração, o que faz a empresa mudar as estratégias de ação sobre eles (PURDY, 2010).

Avalia-se que a oferta de um produto de material reciclado pode trazer clientes de produtos sustentáveis para manter ou aumentar a demanda. A avaliação do preço de venda leva em consideração a percepção de valor do consumidor (ESSOUSSI, 2010; IBM, 2020) frente a um produto considerado sustentável, ou que possui material reciclado, de forma diferente de um produto tradicional.

O risco referente a concorrência pode ser evitado ou transferido, visto que o produto atual é fornecido pela indústria gráfica e de embalagens. O material da embalagem, já produzido pela empresa pode ser vendido, como uma resposta de exploração do risco. O projeto gera um resíduo reciclável, porém não degradável como no caso do produto atual que é o papelão. Sob o ponto de vista ambiental, apesar dos pontos positivos no início do ciclo de vida do produto ao final este pode ter impactos negativos. Considerando o valor sustentável, o projeto deve ser viável nas três dimensões da sustentabilidade. Dado o aumento da vida útil 
do material com o reaproveitamento de resíduos de outras empresas, a empresa está contribuindo com a economia circular, nos aspectos ambiental e também econômico, na redução dos custos da cadeia, que são evitados da disposição em aterro sanitário.

A avaliação dos riscos do projeto evidencia o conhecimento dos riscos internos e externos envolvidos. A quantificação dos riscos permitiu verificar a viabilidade econômica do projeto e o levantamento de propostas de tratamento aos riscos envolvidos. O estudo do gerenciamento de riscos do projeto pode auxiliar a empresa na tomada de decisão sobre a comercialização de seus produtos. Este aponta as ameaças e oportunidades através de técnicas que permitem quantificar os riscos e a geração de valor do negócio na cadeia.

\section{REFERÊNCIAS}

BOCKEN, N., SHORT, S., RANA, P., \& EVANS, S. A literature and practice review to develop sustainable business model archetypes. Journal of Cleaner Production, 65, 42-56. 2014.

BRASIL, Ministério do Meio Ambiente. Plano Nacional de Resíduos Sólidos. 2020. Acesso em: https://www.gov.br/pt-br/noticias/meio-ambiente-e-clima/2020/08/aberta-consulta-publica-sobre-plano-nacionalde-residuos-solidos.

CAGLIANO, A.C.; GRIMALDI, S.; RAFELE, C. Choosing project risk management techniques. A theoretical framework, Journal of Risk Research, 18:2, 232-248, 2015. DOI: 10.1080/13669877.2014.896398

CNI (Confederação Nacional da Indústria); SEBRAE-SP. Publicação: Inovar é Desenvolver a Indústria do Futuro: 30 Casos de Inovação de Pequenas, Médias e Grandes Empresas. 2019.Disponível em: https://www.portaldaindustria.com.br/publicacoes/2019/8/30-casos-de-inovacao-de-pequenas-medias-e-grandesempresas-capitulos-selecionados/\#ambiente-verde $\% 20$

ESSOUSSI, L.H.; LINTON, J.D. New or recycled products: how much are consumers willing to pay? Journal of Consumer Marketing, $27 / 5$ (2010), 458-468. DOI 10.1108/07363761011063358

HART, S.L., MILSTEIN, M.B.M. Creating sustainable value. Academy of Management Executive. Vol. 17, No. 2, p.56-69. 2003.

LEWANDOWSKI, M. Designing the Business Models for Circular Economy-Towards the Conceptual Framework. Sustainability, 8, 43. 2016 doi:10.3390/su8010043.

IBM Institute for Business Value. Haller, K; Lee, J.; Cheung, J. Meet the 2020 consumers driving change - Why brands must deliver on omnipresence, agility, and sustainability. IBM Report (June 2020). Available at: https://www.ibm.com/thought-leadership/institute-business-value/report/consumer-2020

PALOUSIS, N.; LUONG, L.; ABHARY, K. Sustainability risk identification in product development. International Journal of Sustainable Engineering, 3:2, 70-80, 2010. DOI: 10.1080/19397031003686900

PATALA, S. et al. Sustainable value propositions: Framework and implications for technology suppliers. Industrial Marketing Management, 59 (2016) 144-156, 2016.

PMI (Project Management Institute) A Guide to Project Management Body of Knowledge (PMBOK® Guide) 4th ed. Newtown Square, PA, USA. 2008.

PURDY, G. ISO 31000:2009-Setting a New Standard for Risk Management. Risk Analysis, Vol. 30, No. 6, 2010. DOI: 10.1111/j.1539-6924.2010.01442.x

SCHILLER, F.; PRPICH, G. Learning to organise risk management in organisations: what future for enterprise risk management? Journal of Risk Research, Vol. 17, No. 8, 999-1017, 2014. 
SCHULTE, J. HALLSTEDT, S. I. Company Risk Management in Light of the Sustainability Transition. Sustainability 2018, 10, 4137. 2018 